Discrete Comput Geom 35:287-300 (2006)

DOI: $10.1007 / \mathrm{s} 00454-005-1230-\mathrm{y}$

\title{
Common Tangents to Spheres in $\mathbb{R}^{3}$
}

\author{
Ciprian Borcea, ${ }^{1}$ Xavier Goaoc, ${ }^{2}$ Sylvain Lazard, ${ }^{2}$ and Sylvain Petitjean ${ }^{2}$ \\ ${ }^{1}$ Mathematics Department, Rider University, \\ Lawrenceville, NJ 08648, USA \\ borcea@rider.edu \\ ${ }^{2}$ LORIA - INRIA Lorraine, CNRS, and Université Nancy 2, \\ 54602 Villers-lès-Nancy cedex, France \\ \{goaoc, lazard, petitjea\}@loria.fr
}

\begin{abstract}
We prove that four spheres in $\mathbb{R}^{3}$ have infinitely many real common tangents if and only if they have aligned centers and at least one real common tangent.
\end{abstract}

\section{Introduction}

A major issue in geometric computing is to handle degenerate inputs properly in order to design robust algorithms. This often requires recognizing such an input to begin with. In three-dimensional visibility problems, which are ubiquitous in computer graphics and image synthesis, objects with a set of common tangents of improper dimension constitute degenerate configurations. In this paper we determine all degenerate configurations of four distinct spheres, that is, all configurations of four spheres with infinitely many common tangents.

The study of real lines tangent to basic geometric objects has been very active in recent years. This topic includes two closely related directions of research, namely, the characterization of degenerate configurations and the enumeration of lines satisfying geometric constraints. Usually, these problems are approached by studying the degeneracies and counting the number of solutions of some specific polynomial system. The difficulty often resides in eliminating imaginary solutions, solutions at infinity, and components of positive dimension of solutions in order to retain only real affine solutions.

The case of lines tangent to spheres has been persistently investigated. Macdonald et al. [4] proved that four unit spheres have at most 12 common tangents in general, and infinitely many common tangents if and only if the centers are aligned. The bound of 12 was independently obtained by Devillers et al. [2]. Examples show that, in the finite case, 

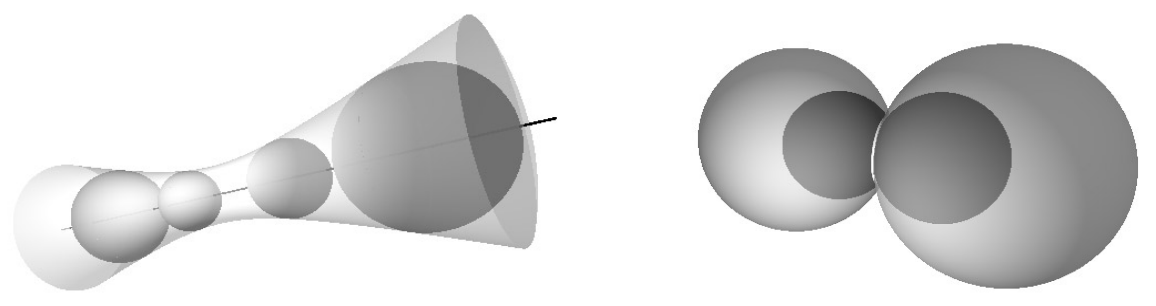

Fig. 1. Two examples of quadruples of spheres with infinitely many common tangents.

this bound is tight [2], [4], yet, according to Megyesi [5], it drops to eight in the case of unit spheres with coplanar but non-collinear centers. However, the upper bound of 12 remains valid when the spheres have arbitrary radii. Sottile and Theobald [8] proved that there are $3 \cdot 2^{n-1}$ complex common tangent lines to $2 n-2$ general spheres in $\mathbb{R}^{n}$, and that there exists a choice of spheres with all common tangents real.

Recently, progress has also been made in understanding the varieties of common tangents to spheres and transversals to lines. Theobald [9] described the configurations of three lines and a sphere having infinitely many common tangents/transversals. Next, Megyesi et al. [7] characterized the families of two lines and two quadrics of $\mathbb{P}^{3}(\mathbb{C})$ with infinitely many tangents/transversals, and applied their results to the case of two lines and two spheres of $\mathbb{R}^{3}$. Last, Megyesi and Sottile [6] classified the families of one line and three spheres of $\mathbb{R}^{3}$ with infinitely many tangents/transversals.

The question of characterizing the positions of four spheres of various radii with infinitely many common tangents remained open. Quoting Theobald [9]: "We conjecture that there does not exist any configuration with four balls of arbitrary radii, non-collinear centers and infinitely many common tangent lines." In this paper we confirm this expectation and prove

Theorem 1. Four distinct spheres in $\mathbb{R}^{3}$ have infinitely many real common tangent lines if and only if they have aligned centers and at least one real common tangent.

More precisely we prove that four spheres with infinitely many common real tangents either intersect in a circle, possibly degenerating to a point, or each sphere has a circle of tangency with one and the same quadric of revolution with symmetry axis the line through all centers (see Fig. 1); such a quadric is unique and can be a cone, a cylinder, or a hyperboloid of one sheet. Furthermore, the common tangents to the four spheres are exactly the common tangents to any three of them.

After introducing some notations and preliminaries in Section 2, we treat the case of four spheres with affinely independent centers in Section 3. Next, we handle in Section 4 the more intricate case of spheres with coplanar centers, no three aligned. Section 5 ends the proof of Theorem 1 with the case of three aligned centers. We obtain, at the same time, the algebraic and semi-algebraic conditions on radii and mutual distances between centers, which characterize four spheres with infinitely many common real tangents. 


\section{Preliminaries}

Notations. Our proofs use points and vectors from $\mathbb{R}^{n}$ and from the real and complex projective spaces of dimension $n, \mathbb{P}^{n}(\mathbb{R})$ and $\mathbb{P}^{n}(\mathbb{C})$. We make no distinction between a point $p$ and the vector from the origin of the frame to $p$. For more clarity, we denote an element of $\mathbb{R}^{n}$ by $\left(a_{1}, \ldots, a_{n}\right)$, and an element of $\mathbb{P}^{n}(\mathbb{R})$ or $\mathbb{P}^{n}(\mathbb{C})$ by $\left(a_{1}: \cdots: a_{n+1}\right)$.

For any two vectors $a, b$ of $\mathbb{R}^{n}, \mathbb{P}^{n}(\mathbb{R})$, or $\mathbb{P}^{n}(\mathbb{C})$, we denote by $a \cdot b$ their dot product, by $a \times b$ their cross product, and by $|a|^{2}$ the dot product $a \cdot a$ (note that $|a|^{2}$ is not the square of the norm of $a$ when $a$ has imaginary coordinates).

Let $\mathcal{S}_{i}$ denote the sphere of $\mathbb{R}^{3}$ with center $c_{i}$ and radius $r_{i}>0$, for $i=1, \ldots, 4$, and let $\left(e_{1}, e_{2}, e_{3}\right)$ be an orthonormal frame of $\mathbb{R}^{3}$. Without loss of generality, we assume that $c_{1}$ is the origin of our frame. The axis of a set of spheres with aligned centers is the line going through these centers.

Tangents to Four Spheres. We begin by reviewing the description of the common tangent lines to four spheres as solutions of a polynomial system, as in [4]. We represent a line in $\mathbb{R}^{3}$ by its closest point to the origin $p \in \mathbb{R}^{3}$ and its direction vector $v \in \mathbb{P}^{2}(\mathbb{R})$. Let $M$ denote the matrix $\left[c_{2}, c_{3}, c_{4}\right]^{T}$ and let $\Phi_{0}$ and $\Phi_{2}(v)$ be the vectors

$$
\Phi_{0}=\left(\begin{array}{l}
\left|c_{2}\right|^{2}+r_{1}^{2}-r_{2}^{2} \\
\left|c_{3}\right|^{2}+r_{1}^{2}-r_{3}^{2} \\
\left|c_{4}\right|^{2}+r_{1}^{2}-r_{4}^{2}
\end{array}\right), \quad \Phi_{2}(v)=-\left(\begin{array}{c}
\left(c_{2} \cdot v\right)^{2} \\
\left(c_{3} \cdot v\right)^{2} \\
\left(c_{4} \cdot v\right)^{2}
\end{array}\right)
$$

Lemma 1. The lines tangent to the four spheres $\mathcal{S}_{1}, \ldots, \mathcal{S}_{4}$ are the common solutions $(p, v)$ in $\mathbb{R}^{3} \times \mathbb{P}^{2}(\mathbb{R})$ of the equations

$$
\begin{gathered}
p \cdot v=0, \\
|p|^{2}=r_{1}^{2}, \\
2|v|^{2} M p=\Phi_{2}(v)+|v|^{2} \Phi_{0} .
\end{gathered}
$$

Proof. A couple $(p, v) \in \mathbb{R}^{3} \times \mathbb{P}^{2}(\mathbb{R})$ represents a line if and only if (1) is satisfied. A line $(p, v)$ is tangent to sphere $\mathcal{S}_{i}$ if and only if its squared distance to $c_{i}$ is $r_{i}^{2}$, that is, if and only if

$$
\left|\left(c_{i}-p\right) \times v\right|^{2}=r_{i}^{2}|v|^{2} .
$$

Expanding this equation yields

$$
\left|c_{i} \times v\right|^{2}+|p \times v|^{2}-2\left(c_{i} \times v\right) \cdot(p \times v)=r_{i}^{2}|v|^{2} .
$$

Applying to $\left(c_{i} \times v\right) \cdot(p \times v)$ the scalar triple product identity $a \cdot(b \times c)=b \cdot(c \times a)$, then the vector triple product identity $a \times(b \times c)=(a \cdot c) b-(a \cdot b) c$, and finally using (1) we get

$$
(p \times v) \cdot\left(c_{i} \times v\right)=c_{i} \cdot(v \times(p \times v))=c_{i} \cdot((v \cdot v) p-(v \cdot p) v)=|v|^{2} c_{i} \cdot p .
$$

Since $p$ and $v$ are orthogonal, $|p \times v|^{2}=|p|^{2}|v|^{2}$ and thus (4) becomes

$$
2|v|^{2} c_{i} \cdot p=\left|c_{i} \times v\right|^{2}+|v|^{2}\left(|p|^{2}-r_{i}^{2}\right) .
$$


As $\left|c_{i} \times v\right|^{2}+\left(c_{i} \cdot v\right)^{2}=\left|c_{i}\right|^{2}|v|^{2}$, we finally get that

$$
2|v|^{2} c_{i} \cdot p=-\left(c_{i} \cdot v\right)^{2}+|v|^{2}\left(\left|c_{i}\right|^{2}+|p|^{2}-r_{i}^{2}\right) .
$$

Equation (5) for $i=1$ is equivalent to (2) since $c_{1}$ is the origin of the frame. It follows that the four equations (5) for $i=1, \ldots, 4$ are equivalent to the two equations (2) and (3).

The approach used to show that infinitely many tangent lines to spheres can only happen when the centers of the spheres are aligned is as follows. We eliminate $p$ among (1)-(3), giving two curves ${ }^{1}$ in the two-dimensional projective space of directions, whose intersection contains all directions along which a common tangent line to the four spheres is observed. We then prove that, when the centers are non-collinear, the two curves intersect in a finite number of points.

The key idea behind the proofs of Section 3 (affinely independent centers) and Section 4 (coplanar centers) is that if the two curves, envisaged as complex projective curves, had a common component of positive dimension, this component would intersect the imaginary conic $|v|^{2}=0$ and we show that this is not the case. Intersecting the curve with $|v|^{2}=0$ is inspired by the relation of the Grassmannian of lines in $\mathbb{P}^{3}(\mathbb{C})$ with the $(p, v)$ coordinate system, well adapted to the representation of lines in the affine part $\mathbb{R}^{3} \subset \mathbb{P}^{3}(\mathbb{R})$.

It should be stressed that any solution to the problem of characterizing sets of four spheres with infinitely many tangent lines must be computational to some extent, because while we are interested in real lines, the "native" system of equations is over $\mathbb{C}$. Any understanding of the system should involve sensitivity to complex degeneracies. In our proof, computations flow towards revealing such complex degeneracies, but are shortcircuited by use of reality assumptions.

\section{Affinely Independent Centers}

We first investigate the case of spheres with affinely independent centers.

Proposition 1. Four spheres with affinely independent centers have at most 12 common tangent lines.

Proof. First note that matrix $M$ is invertible since the spheres have affinely independent centers. Considering $(p, v)$ in $\mathbb{R}^{3} \times \mathbb{P}^{2}(\mathbb{R})$, we have $|v|^{2} \neq 0$ and thus (1)-(3) are equivalent to the three equations

$$
\begin{gathered}
p=M^{-1}\left(\frac{\Phi_{2}(v)}{2|v|^{2}}+\frac{1}{2} \Phi_{0}\right), \\
\left(M^{-1}\left(\Phi_{2}(v)+|v|^{2} \Phi_{0}\right)\right) \cdot v=0, \\
\left|M^{-1}\left(\Phi_{2}(v)+|v|^{2} \Phi_{0}\right)\right|^{2}=4 r_{1}^{2}|v|^{4} .
\end{gathered}
$$

\footnotetext{
${ }^{1} \mathrm{~A}$ cubic and a quartic when the centers are affinely independent, a conic and a sextic when the centers are coplanar with no three aligned.
} 
Equation (6) expresses the point $p$ in terms of the direction vector $v$, proving that there is at most one line tangent to the four spheres with a given direction. The remaining equations are a cubic (7) and a quartic (8) in $v$, and their intersection represents the directions $v \in \mathbb{P}^{2}(\mathbb{R})$ along which there is a tangent to the four spheres. We want to prove that the cubic and the quartic intersect in at most 12 points in $\mathbb{P}^{2}(\mathbb{R})$. For that purpose we prove this property in $\mathbb{P}^{2}(\mathbb{C})$, by contradiction.

If the cubic and the quartic have in $\mathbb{P}^{2}(\mathbb{C})$ a common component of positive dimension, this component intersects the conic $|v|^{2}=0$; this is a property of any two curves in $\mathbb{P}^{2}(\mathbb{C})$ which does not dispute the fact that the real solutions of (6)-(8) satisfy $|v|^{2} \neq 0$. We now prove that the intersection in $\mathbb{P}^{2}(\mathbb{C})$ of the cubic $(7)$, the quartic (8) and the conic $|v|^{2}=0$ is empty. This system simplifies to

$$
\left\{\begin{array}{l}
|v|^{2}=0 \\
\left(M^{-1} \Phi_{2}(v)\right) \cdot v=0 \\
\left|M^{-1} \Phi_{2}(v)\right|^{2}=0
\end{array}\right.
$$

The first two equations express the fact that $M^{-1} \Phi_{2}(v)$ is on the tangent at $v$ to the smooth conic $|v|^{2}=0$, and the last that $M^{-1} \Phi_{2}(v)$ is itself on that conic. It follows that $M^{-1} \Phi_{2}(v)$ and $v$ are one and the same projective point. Thus there exists $\mu \neq 0$ in $\mathbb{C}$ such that

$$
M^{-1} \Phi_{2}(v)=\mu v, \quad \text { that is } \quad \Phi_{2}(v)=\mu M v .
$$

Expanding this last equality yields $-\left(c_{i} \cdot v\right)^{2}=\mu c_{i} \cdot v$, for $i=2, \ldots, 4$, which implies that every term $c_{i} \cdot v$ is 0 or $-\mu$. This leads to

$$
M v=-\mu\left(\begin{array}{l}
a_{2} \\
a_{3} \\
a_{4}
\end{array}\right),
$$

where each $a_{i}$ is equal to 0 or 1 . Let $a$ denote the vector of the $a_{i}$. Plugging $v=\mu M^{-1} a$ in the equation of the conic $|v|^{2}=0$ yields

$$
\mu^{2}\left|M^{-1} a\right|^{2}=0
$$

The vector $M^{-1} a$ is real, thus $\mu=0$ or $a=0$. In both cases, (9) implies $v=0$. Thus there is no common solution in $\mathbb{P}^{2}(\mathbb{C})$ for the system of the conic, the cubic, and the quartic, hence the cubic (7) and quartic (8) cannot intersect in a curve. By Bezout's theorem, they intersect in at most 12 points, and since there is at most one line tangent to the four spheres with a given direction by (6), this completes the proof.

\section{Coplanar Centers}

We now treat the more intricate case of four spheres whose centers are coplanar but such that no three centers are aligned.

Proposition 2. Four spheres with coplanar centers, no three aligned, have at most 12 common tangents. 
Let $(p, v) \in \mathbb{R}^{3} \times \mathbb{P}^{2}(\mathbb{R})$ represent a line tangent to the four spheres $\mathcal{S}_{1}, \ldots, \mathcal{S}_{4}$. By Lemma $1,(p, v)$ is a solution of (1)-(3). As in Section 3, we start by extracting from these equations two equations in $v$.

Without loss of generality, we may assume that the sphere centers span the plane $\left(e_{1}, e_{2}\right)$ :

$$
M=\left(\begin{array}{lll}
c_{21} & c_{22} & 0 \\
c_{31} & c_{32} & 0 \\
c_{41} & c_{42} & 0
\end{array}\right) .
$$

Let $M_{12}$ be the $2 \times 2$ upper left sub-matrix of $M$, which is invertible since no three centers are aligned. For any vector $a$, let $a_{12}$ be the vector that consists of the first two rows of $a$ and let $a_{3}$ be its third row.

We first assume that $v_{3} \neq 0$. It follows from $p \cdot v=0$ that

$$
p_{3}=-\frac{p_{12} \cdot v_{12}}{v_{3}}
$$

and $p_{12}$ is characterized using (3):

$$
2|v|^{2} p_{12}=M_{12}^{-1}\left(\left(\Phi_{2}(v)\right)_{12}+|v|^{2}\left(\Phi_{0}\right)_{12}\right) .
$$

Let $\Psi_{2}(v)=M_{12}^{-1}\left(\Phi_{2}(v)\right)_{12}$ and $\Psi_{0}=M_{12}^{-1}\left(\Phi_{0}\right)_{12}$. As $\Phi_{2}(v)$ and $\Psi_{2}(v)$ do not depend on $v_{3}$, we may write them as $\Phi_{2}\left(v_{12}\right)$ and $\Psi_{2}\left(v_{12}\right)$. Then

$$
2|v|^{2} p_{12}=\Psi_{2}\left(v_{12}\right)+|v|^{2} \Psi_{0}
$$

Substituting the expression of $p_{3}$ from (10) in (2) gives

$$
\left|p_{12}\right|^{2}+\left(\frac{p_{12} \cdot v_{12}}{v_{3}}\right)^{2}-r_{1}^{2}=0
$$

Then multiplying by $4|v|^{4} v_{3}^{2}$ and substituting $2|v|^{2} p_{12}$ by its expression from (11) gives the following sextic equation in $v$ :

$$
\left.\left.v_{3}^{2}\left|\Psi_{2}\left(v_{12}\right)+\right| v\right|^{2} \Psi_{0}\right|^{2}+\left(\left(\Psi_{2}\left(v_{12}\right)+|v|^{2} \Psi_{0}\right) \cdot v_{12}\right)^{2}-4|v|^{4} v_{3}^{2} r_{1}^{2}=0 .
$$

For any $p, q$ in $\mathbb{P}^{3}(\mathbb{C})$, we have, by transposition:

$$
(M p) \cdot q=p \cdot\left(M^{T} q\right) .
$$

Let $\omega$ be a non-zero kernel vector of $M^{T}$. Then $(M p) \cdot \omega=p \cdot\left(M^{T} \omega\right)=0$. Substituting the expression of $M p$ from (3), we obtain that $v$ must be on the following conic:

$$
\Phi_{2}\left(v_{12}\right) \cdot \omega+|v|^{2} \Phi_{0} \cdot \omega=0 .
$$

Notice that (12) and (13), obtained for $v_{3} \neq 0$, are still valid for $v_{3}=0$ by continuity. We thus get the following lemma.

Lemma 2. The direction $v \in \mathbb{P}^{2}(\mathbb{R})$ of a line tangent to the four spheres $\mathcal{S}_{1}, \ldots, \mathcal{S}_{4}$ satisfies the sextic (12) and the conic (13). 
Lemma 3. If the sextic (12) and the conic (13) admit a component of positive dimension of common solutions in $\mathbb{P}^{2}(\mathbb{C})$, then it intersects the conic $|v|^{2}=0$ and any point $v$ in the intersection satisfies

$$
\begin{gathered}
\exists \lambda \in \mathbb{C}, \quad \Psi_{2}\left(v_{12}\right)=\lambda v_{12}, \\
\Phi_{2}\left(v_{12}\right) \cdot \omega=0 .
\end{gathered}
$$

Proof. If (12) and (13) share a component of positive dimension in $\mathbb{P}^{2}(\mathbb{C})$, then this component, seen as a curve of $\mathbb{P}^{2}(\mathbb{C})$, intersects the conic $|v|^{2}=0$. Let $v \in \mathbb{P}^{2}(\mathbb{C})$ be in this intersection. Then (13) becomes (15). Now, it follows from $|v|^{2}=0$ that $v_{3}^{2}=-\left|v_{12}\right|^{2}$, and thus (12) becomes

$$
-\left|v_{12}\right|^{2}\left|\Psi_{2}\left(v_{12}\right)\right|^{2}+\left(\Psi_{2}\left(v_{12}\right) \cdot v_{12}\right)^{2}=0 .
$$

Since $|x|^{2}|y|^{2}-(x \cdot y)^{2}=\operatorname{det}(x, y)^{2}$ for any $x, y \in \mathbb{C}^{2}$, the equation is equivalent to $\operatorname{det}\left(v_{12}, \Psi_{2}\left(v_{12}\right)\right)=0$ which is equivalent to (14) ( $v$ is on $|v|^{2}=0$ so we cannot have $\left.v_{12}=0\right)$.

In the following we consider the centers $c_{1}=0, c_{2}, c_{3}, c_{4}$ as two-dimensional points (i.e., we forget the third coordinate, which is 0 ). For any vector $x \in \mathbb{R}^{2}$ we denote by $x^{\perp}$ its orthogonal vector obtained by a rotation of angle $\pi / 2$.

Lemma 4. If (14) and (15) have a common solution $v_{12}$ in $\mathbb{P}^{1}(\mathbb{C})$, it must satisfy $v_{12}=c_{i}^{\perp}$ and $v_{12} \cdot\left(c_{j}-c_{k}\right)=0$, with $\{i, j, k\}=\{2,3,4\}$ (which implies that $c_{1}, c_{2}, c_{3}$, $c_{4}$ are the vertices of a trapezoid).

Proof. From $M^{T} \omega=0$ we get

$$
M^{T} \omega=\left(\begin{array}{cc}
M_{12}^{T} & c_{4} \\
0 & 0
\end{array}\right)\left(\begin{array}{c}
\omega_{12} \\
\omega_{3}
\end{array}\right)=\left(\begin{array}{c}
M_{12}^{T} \omega_{12}+\omega_{3} c_{4} \\
0
\end{array}\right)=0 .
$$

Thus $\omega_{12}=-\omega_{3}\left(M_{12}^{T}\right)^{-1} c_{4}$ and $\omega_{3} \neq 0$ (otherwise, $\omega_{12}=0$ thus $\omega=0$ contradicting its definition). Now, we can write $(15)$ as $\left(\Phi_{2}\left(v_{12}\right)\right)_{12} \cdot \omega_{12}-\left(c_{4} \cdot v_{12}\right)^{2} \omega_{3}=0$, and substituting our expression of $\omega_{12}$ yields

$$
-\omega_{3}\left(\Phi_{2}\left(v_{12}\right)\right)_{12} \cdot\left(\left(M_{12}^{T}\right)^{-1} c_{4}\right)-\left(c_{4} \cdot v_{12}\right)^{2} \omega_{3}=0,
$$

which simplifies, by transposition, into

$$
\left(M_{12}^{-1}\left(\Phi_{2}\left(v_{12}\right)\right)_{12}\right) \cdot c_{4}+\left(c_{4} \cdot v_{12}\right)^{2}=0 .
$$

Hence, an equivalent expression for (15) is

$$
\Psi_{2}\left(v_{12}\right) \cdot c_{4}+\left(c_{4} \cdot v_{12}\right)^{2}=0 .
$$

Substituting $\Psi_{2}\left(v_{12}\right)=\lambda v_{12}$ from (14) into (16) leads to

$$
\left(c_{4} \cdot v_{12}\right)^{2}=-\lambda c_{4} \cdot v_{12} .
$$


By a similar reasoning, we can express the conic (15) using $c_{2}$ or $c_{3}$ in expressions similar to (16), and the above argument yields that

$$
\left(c_{i} \cdot v_{12}\right)^{2}=-\lambda c_{i} \cdot v_{12}, \quad i=2,3,4 .
$$

If $c_{i} \cdot v_{12} \neq 0$ for $i=2,3$, and 4 then $\left(c_{2}-c_{3}\right) \cdot v_{12}=\left(c_{2}-c_{4}\right) \cdot v_{12}=0$ and, since $c_{2}, c_{3}$, and $c_{4}$ are not aligned, $v_{12}=0$ contradicting $v_{12} \in \mathbb{P}^{1}(\mathbb{C})$. Hence, $v_{12}$ must be orthogonal to some $c_{i}, i \in\{2,3,4\}$. Since $v_{12} \in \mathbb{P}^{1}(\mathbb{C})$, we can assume that $v_{12}=c_{i}^{\perp}$. Since no three centers are aligned, $v_{12}$ is orthogonal to neither $c_{j}$ nor $c_{k}$, with $\{i, j, k\}=\{2,3,4\}$. Thus (17) yields

$$
-\lambda=c_{j} \cdot c_{i}^{\perp}=c_{k} \cdot c_{i}^{\perp}, \quad \text { and so } \quad c_{i}^{\perp} \cdot\left(c_{j}-c_{k}\right)=0 .
$$

This means that the segments $c_{1} c_{i}$ and $c_{j} c_{k}$ are parallel and thus the centers of the spheres are the vertices of a trapezoid.

Lemma 5. If the sextic (12) and the conic (13) have a common component of positive dimension in $\mathbb{P}^{2}(\mathbb{C}),(14)$ and $(15)$ have at least two distinct solutions in $\mathbb{P}^{1}(\mathbb{C})$.

Proof. Assume that the sextic (12) and the conic (13) share a component of positive dimension. Then by Lemmas 3 and 4, (14) and (15) admit a common solution $v_{12}=c_{i}^{\perp}$ for $i=2,3$, or 4 . By relabeling if necessary, we can assume $v_{12}=c_{4}^{\perp}$. Suppose, for a contradiction, that $c_{4}^{\perp}$ is the unique common solution of (14) and (15).

By Lemma 3, any point in the intersection of the conic $|v|^{2}=0$ and the common component of the sextic (12) and the conic (13) satisfies (14) and (15). Thus any such point satisfies $v_{12}=c_{4}^{\perp}$ and $|v|^{2}=0$, and is equal to one of the two points of coordinates $\left(c_{4}^{\perp}: \pm i\left|c_{4}\right|\right)$. Hence the common component contains at least one of these two points.

The common component of the sextic (12) and the conic (13) is either the conic itself or a line. In the latter case the equation of the line is real because otherwise its conjugate is also contained in the conic and in the sextic (since their equations are real); the sextic then contains the conic, which corresponds to the first case. Hence the equation of the common component is real in both cases. Thus, since the common component contains one of the two points $\left(c_{4}^{\perp}: \pm i\left|c_{4}\right|\right)$, it also contains its conjugate, hence the two points.

We now discard the case where the common component is the conic by deriving a contradiction with our assumption that no three centers are collinear. If the conic is contained in the sextic, it meets $|v|^{2}=0$ in the two points $\left(c_{4}^{\perp}: \pm i\left|c_{4}\right|\right)$, which are therefore tangency points. This means that (16), which is our conic modulo $|v|^{2}=0$, has a double root at $v_{12}=c_{4}^{\perp}$. Since any degree-two polynomial in $v_{12} \in \mathbb{P}^{1}(\mathbb{C})$ that has $c_{4}^{\perp}$ as double root is proportional to $\left(c_{4} \cdot v_{12}\right)^{2}$, we get that

$$
\Psi_{2}\left(v_{12}\right) \cdot c_{4}=\alpha\left(c_{4} \cdot v_{12}\right)^{2}
$$

for some $\alpha \in \mathbb{C}$ and all $v_{12} \in \mathbb{P}_{1}(\mathbb{C})$. Computing $\operatorname{det}\left(M_{12}\right) M_{12}^{-1}$ gives the matrix with columns $\left[-c_{3}^{\perp} c_{2}^{\perp}\right]$, thus our equation becomes

$$
\Psi_{2}\left(v_{12}\right) \cdot c_{4}=\frac{1}{\operatorname{det}\left(M_{12}\right)}\left[\left(c_{3}^{\perp} \cdot c_{4}\right)\left(c_{2} \cdot v_{12}\right)^{2}-\left(c_{2}^{\perp} \cdot c_{4}\right)\left(c_{3} \cdot v_{12}\right)^{2}\right]=\alpha\left(c_{4} \cdot v_{12}\right)^{2} .
$$


Since the four centers form a trapezoid we have $c_{4}=v\left(c_{2}-c_{3}\right)$ for some $v \in \mathbb{R}^{3}$. Replacing $c_{4}$ by its expression and simplifying by factor $c_{3}^{\perp} \cdot c_{2}=-c_{2}^{\perp} \cdot c_{3}$ yields

$$
\left(c_{2} \cdot v_{12}\right)^{2}-\left(c_{3} \cdot v_{12}\right)^{2}=\kappa\left(\left(c_{2}-c_{3}\right) \cdot v_{12}\right)^{2},
$$

for some $\kappa \in \mathbb{C}$. Writing $v_{12}=x c_{2}^{\perp}+y c_{3}^{\perp}$ we obtain

$$
\left(c_{2} \cdot c_{3}^{\perp}\right)^{2}\left(y^{2}-x^{2}-\kappa(x+y)^{2}\right)=0
$$

for all $(x, y) \in \mathbb{P}^{1}(\mathbb{C})$, which forces the proportionality of $c_{2}$ and $c_{3}$ and their alignment with $c_{1}$. Thus, if no three centers are aligned the conic cannot be contained in the sextic.

Now we examine the second alternative, when the common component of the sextic (12) and the conic (13) is a line. This line contains the two points $\left(c_{4}^{\perp}: \pm i\left|c_{4}\right|\right)$ and thus contains the point $\left(c_{4}^{\perp}: v_{3}\right)$ for all $v_{3} \in \mathbb{C}$. Thus all the coefficients of the sextic (12) viewed as an equation in $v_{3}$ with coefficients depending on $v_{12}=c_{4}^{\perp}$ must vanish. In particular, the constant and the coefficient of $v_{3}^{2}$ minus $\left|c_{4}\right|^{4}$ times the coefficient of $v_{3}^{6}$ both vanish and are equal to

$$
\begin{array}{r}
\Psi_{2}\left(c_{4}^{\perp}\right) \cdot c_{4}^{\perp}+\left|c_{4}\right|^{2} \Psi_{0} \cdot c_{4}^{\perp}=0, \\
\left|\Psi_{2}\left(c_{4}^{\perp}\right)\right|^{2}+2\left|c_{4}\right|^{2} \Psi_{2}\left(c_{4}^{\perp}\right) \cdot \Psi_{0}=0 .
\end{array}
$$

From the proof of Lemma 4, we know that $\Psi_{2}\left(c_{4}^{\perp}\right)=\lambda c_{4}^{\perp}$ with $\lambda=-c_{2} \cdot c_{4}^{\perp}=-c_{3} \cdot c_{4}^{\perp}$. Thus, the relations become

$$
\begin{array}{r}
\left|c_{4}\right|^{2}\left(\lambda+\Psi_{0} \cdot c_{4}^{\perp}\right)=0, \\
\lambda\left|c_{4}\right|^{2}\left(\lambda+2 \Psi_{0} \cdot c_{4}^{\perp}\right)=0 .
\end{array}
$$

Since no three centers are aligned, $\lambda \neq 0$ and $\left|c_{4}\right|^{2} \neq 0$, and these two equations imply $\lambda=0$, a contradiction.

Lemma 6. The sextic (12) and the conic (13) cannot have a component of positive dimension of common solutions.

Proof. Assume that the sextic (12) and the conic (13) have a common component of positive dimension. Lemmas 4 and 5 yield that (14) and (15) then have at least two distinct solutions among $\left\{c_{2}^{\perp}, c_{3}^{\perp}, c_{4}^{\perp}\right\}$. By relabeling the centers, we may assume these solutions are $c_{2}^{\perp}$ and $c_{3}^{\perp}$. Lemma 4 gives that

$$
c_{2}^{\perp} \cdot\left(c_{4}-c_{3}\right)=0 \quad \text { and } \quad c_{3}^{\perp} \cdot\left(c_{4}-c_{2}\right)=0 .
$$

Thus, $c_{2}$ is proportional to $c_{4}-c_{3}$, and $c_{3}$ is proportional to $c_{4}-c_{2}$. Therefore, $c_{2}+c_{3}=c_{4}$ and the centers form a parallelogram. By translating our frame to the center of that parallelogram, we may assume that the centers are at $a=\left(a_{1}, a_{2}, 0\right), b=\left(b_{1}, b_{2}, 0\right)$, $-a$, and $-b$, with corresponding radii $r_{i}, i=1, \ldots, 4$. On occasion, we abuse notation, and allow $a$ and $b$ to stand for $\left(a_{1}, a_{2}\right)$, respectively $\left(b_{1}, b_{2}\right)$.

Subtracting (5) for $i=1$ from its expression for $i=3$ leads to

$$
4(a \cdot p)=r_{3}^{2}-r_{1}^{2},
$$


and the same operation for $i=2$ and $i=4$ yields

$$
4(b \cdot p)=r_{4}^{2}-r_{2}^{2} .
$$

This shows that the first two coordinates $p_{12}$ of $p$ are determined by centers and radii alone, and remain constant. Thus, all the common tangents to the four spheres meet the line perpendicular to the plane of the centers in $p_{12}$.

Megyesi and Sottile [6] address a situation of this nature and show that the common tangents/transversals to three spheres and a line cannot be infinitely many unless the three spheres have collinear centers. We give here an independent proof which continues the above line of thought.

Recall that (12) and (13) were obtained from (1)-(3) by eliminating $p$. Operating "in reverse," it is easy to see that a one-dimensional component of solutions for (12) and (13) would produce a one-dimensional family of solutions for (1)-(3). We now show this cannot happen.

Rewriting (5) for the centers $a$ and $b$ gives

$$
\begin{aligned}
& (a \cdot v)^{2}=|v|^{2}\left(|a|^{2}+|p|^{2}-\frac{1}{2}\left(r_{1}^{2}+r_{3}^{2}\right)\right), \\
& (b \cdot v)^{2}=|v|^{2}\left(|b|^{2}+|p|^{2}-\frac{1}{2}\left(r_{2}^{2}+r_{4}^{2}\right)\right) .
\end{aligned}
$$

Let $\alpha=|a|^{2}-\frac{1}{2}\left(r_{1}^{2}+r_{3}^{2}\right)$ and $\beta=|b|^{2}-\frac{1}{2}\left(r_{2}^{2}+r_{4}^{2}\right)$. Subtracting (19) from (18) gives the conic

$$
\left((a+b) \cdot v_{12}\right)\left((a-b) \cdot v_{12}\right)=|v|^{2}(\alpha-\beta) .
$$

Multiplying (18), (19), and $v_{3}^{2}$ together and dividing by $|v|^{2}$ gives

$$
\left(a \cdot v_{12}\right)^{2}\left(\beta+\left|p_{12}\right|^{2}+p_{3}^{2}\right) v_{3}^{2}=\left(b \cdot v_{12}\right)^{2}\left(\alpha+\left|p_{12}\right|^{2}+p_{3}^{2}\right) v_{3}^{2},
$$

or, equivalently, using (10),

$$
\left(\left|p_{12}\right|^{2} v_{3}^{2}+\left(p_{12} \cdot v_{12}\right)^{2}\right)\left((a+b) \cdot v_{12}\right)\left((a-b) \cdot v_{12}\right)=v_{3}^{2}\left(\alpha\left(b \cdot v_{12}\right)^{2}-\beta\left(a \cdot v_{12}\right)^{2}\right) \text {. }
$$

For the conic (20) and the quartic (21) to have a common one-dimensional component, it is necessary that equality holds for any $v_{12} \in \mathbb{P}^{1}$ and some adequate value(s) for $v_{3}$. Indeed, the projection $v \mapsto v_{12}$ of the common component cannot be constant, for with fixed $v_{12}$ and (already known) fixed $p_{12},(10)$ and (18) (or (19)) would determine only a finite number of solutions $v_{3}$.

Evaluating (20) and (21) at $v_{12}=(a+b)^{\perp}$, we find no possible value for $v_{3}$, unless $\alpha=\beta$. Returning this necessary condition into (20) implies $v_{12}=(a \pm b)^{\perp}$ contradicting the fact that (20) and (21) hold for all $v_{12} \in \mathbb{P}^{1}$.

We now conclude the case of spheres with coplanar centers.

Proof of Proposition 2. By Lemmas 2 and 6, there are finitely many directions along which the spheres have a common tangent. For each such direction $v$, a line tangent to the four spheres projects onto a plane orthogonal to $v$ into a point that lies on the common intersection of the four circles obtained as the boundary of the projection of each sphere. There are thus at most two lines tangent to the four spheres per direction. Hence there are finitely many lines tangent to the four spheres. Now, the bound of 12 directly follows from the non-coplanar case (Proposition 1) by continuity. 


\section{Collinear Centers}

In this section we conclude the proof of Theorem 1. We first establish the following lemma.

Lemma 7. The common tangents to three distinct spheres with collinear centers and no common intersection are, if any, the ruling $(s)$ of a single quadric of revolution with symmetry axis the line through all centers. This quadric can be a cone, a cylinder, or a hyperboloid of one sheet.

Proof. Suppose that three distinct spheres with collinear centers admit a common tangent. Such a tangent is not orthogonal to the axis of the three spheres since they have no common intersection. Furthermore, such a tangent remains tangent after a rotation about this axis. Thus the common tangents to the three spheres are the rulings of a collection $\mathcal{Q}$ of quadrics of revolution with symmetry axis the line through all centers (see Fig. 1); these quadrics have to be cylinders, cones, or hyperboloids of one sheet. Assume for a contradiction that $\mathcal{Q}$ consists of more than one quadric.

We take the line through the centers to be the $y$-axis in some $(x, y)$-plane. This plane intersects the quadrics of $\mathcal{Q}$ into a collection $\mathcal{C}$ of conics symmetric with respect to the $y$-axis which have equations of the following form:

$$
x^{2}+A y^{2}+B y+C=0, \quad A \leq 0, \quad B^{2}-4 A C \leq 0
$$

The $(x, y)$-plane also intersects the three spheres into three circles, with centers $\left(0, \alpha_{i}\right)$ and radii $r_{i}, i=1, \ldots, 3$, that are tangent to the conics of $\mathcal{C}$. Since these conics and circles are symmetric with respect to the $y$-axis, two of them are tangent if and only if they intersect in exactly two points with the same $y$-coordinate. Thus a conic (22) and a circle of center $\left(0, \alpha_{i}\right)$ and radius $r_{i}$ are tangent if and only if

$$
\left(x^{2}+A y^{2}+B y+C\right)-\left(x^{2}+\left(y-\alpha_{i}\right)^{2}-r_{i}^{2}\right)=0
$$

has a double solution in $y$, i.e., the discriminant vanishes:

$$
\delta_{i}=\left(B+2 \alpha_{i}\right)^{2}-4(A-1)\left(C+r_{i}^{2}-\alpha_{i}^{2}\right)=0
$$

For the three circles, this gives a system of three equations in the three indeterminates $(A, B, C)$. This system is linear in $C$ (with a non-zero coefficient since $A \leq 0$ ) and thus has more than one solution only if the linear system in $(A, B)$,

$$
\left\{\begin{array}{l}
\delta_{1}-\delta_{2}=\left(\left(\alpha_{1}^{2}-\alpha_{2}^{2}\right)-\left(r_{1}^{2}-r_{2}^{2}\right)\right) A+\left(\alpha_{1}-\alpha_{2}\right) B+r_{1}^{2}-r_{2}^{2}=0 \\
\delta_{1}-\delta_{3}=\left(\left(\alpha_{1}^{2}-\alpha_{3}^{2}\right)-\left(r_{1}^{2}-r_{3}^{2}\right)\right) A+\left(\alpha_{1}-\alpha_{3}\right) B+r_{1}^{2}-r_{3}^{2}=0
\end{array}\right.
$$

does, that is, only if the determinant of the coefficients of $A$ and $B$, and the determinant of the constant coefficients and the coefficients of $B$ both vanish. The sum of these determinants also vanishes and is equal to

$$
\left|\begin{array}{ll}
\alpha_{1}^{2}-\alpha_{2}^{2} & \alpha_{1}-\alpha_{2} \\
\alpha_{1}^{2}-\alpha_{3}^{2} & \alpha_{1}-\alpha_{3}
\end{array}\right|=\left(\alpha_{1}-\alpha_{2}\right)\left(\alpha_{1}-\alpha_{3}\right)\left(\alpha_{2}-\alpha_{3}\right)
$$


Hence at least two centers are equal which implies that one sphere is strictly contained in another. The three spheres thus have no common tangent, a contradiction.

Remark. Actually solving the system (23), $i=1,2,3$, yields, in terms of radii and oriented distances between centers $d_{i j}=\alpha_{j}-\alpha_{i}$ :

$$
\begin{aligned}
A= & \frac{1}{D}\left(r_{1}^{2} d_{23}+r_{2}^{2} d_{31}+r_{3}^{2} d_{12}\right) \\
B^{2}-4 A C= & \frac{-1}{d_{23} d_{31} d_{12} D}\left(r_{1} d_{23}+r_{2} d_{31}+r_{3} d_{12}\right)\left(r_{1} d_{23}+r_{2} d_{31}-r_{3} d_{12}\right) \\
& *\left(r_{1} d_{23}-r_{2} d_{31}+r_{3} d_{12}\right)\left(-r_{1} d_{23}+r_{2} d_{31}+r_{3} d_{12}\right)
\end{aligned}
$$

where $D=d_{23} d_{31} d_{12}+r_{1}^{2} d_{23}+r_{2}^{2} d_{31}+r_{3}^{2} d_{12}$.

We can now prove Theorem 1.

Proof of Theorem 1. Consider four distinct spheres with infinitely many real common tangents. By Propositions 1 and 2, the centers of at least three of the spheres are aligned.

If these three spheres intersect in a circle, their common tangents are the tangents to that circle in its plane. To be tangent to infinitely many of these lines, the fourth sphere has to contain that circle (and, if that circle is degenerate to a point, the four spheres must have the same tangent plane at this point). Thus all four spheres have aligned centers.

If the three spheres with aligned centers do not have a common intersection, then by Lemma 7 their common tangents are the rulings of a single quadric having their axis as axis of revolution. To be tangent to infinitely many lines contained in this quadric, the fourth sphere must have its center on the axis of the quadric (and adequate radius as determined below), hence the four spheres have aligned centers.

Conversely, four spheres with aligned centers and at least one common tangent have infinitely many common tangents, by symmetry of revolution. This concludes the proof of Theorem 1 and provides the finer geometric characterization stated in Section 1.

As shown above, four spheres with collinear centers and no common intersection admit infinitely many real common tangents if and only if there exists a conic (22) whose coefficients $A, B, C$ satisfy (23) for all $i=1, \ldots, 4$. These four equations admit a solution if and only if the relation obtained by eliminating $A, B, C$ is satisfied. One can put the result in the permutation invariant form in terms of the oriented distances $d_{i j}=\alpha_{j}-\alpha_{i}$ and the radii $r_{k}$ :

$$
\sum_{k=1}^{4} \frac{r_{k}^{2}}{\prod_{i \neq k} d_{k i}}=0
$$

In order to obtain infinitely many real common tangents, the coefficients $A, B, C$ must also satisfy the semi-algebraic conditions

$$
A \leq 0, \quad B^{2}-4 A C \leq 0
$$


noted in (22). $A$ and $B^{2}-4 A C$ can be obtained in terms of the $d_{i j}$ and $r_{k}$ by solving the system of equations, as illustrated after Lemma 7.

The case of four spheres intersecting in a common circle or tangent in a common point is a limit case of the situation above, and thereby subject to the same algebraic and semi-algebraic conditions.

Remark. When a configuration of four spheres is given in terms of the Cartesian coordinates of the four centers $c_{i}=\left(x_{i}, y_{i}, z_{i}\right)$ and the corresponding radii $r_{i}$, expressing the collinearity of the centers involves quadratic equations in their coordinates, and, in view of

$$
\frac{d_{i j}}{d_{i k}}=\frac{x_{j}-x_{i}}{x_{k}-x_{i}}=\frac{y_{j}-y_{i}}{y_{k}-y_{i}}=\frac{z_{j}-z_{i}}{z_{k}-z_{i}}
$$

and $d_{i j}^{2}=\left(x_{j}-x_{i}\right)^{2}+\left(y_{j}-y_{i}\right)^{2}+\left(z_{j}-z_{i}\right)^{2}$, testing conditions (24) and (25) amounts to evaluating polynomials of degree at most five in the Cartesian coordinates and radii.

\section{Conclusion}

This paper answers a question left open for several years by characterizing the sets of four spheres of various radii with infinitely many common tangent lines. This completes the description of degeneracies for common tangents to spheres in $\mathbb{R}^{3}$.

Some of our results generalize to the case of quadric surfaces. In a companion paper [1] we characterize the families of quadrics in $\mathbb{P}^{3}(\mathbb{C})$ whose common tangents sweep another quadric surface. The result of the present paper appears as a particular case obtained by considering real tangents to real spheres. Extending our characterization to quadruples of quadrics with infinitely many real common tangents remains an open problem.

Results of the kind proved in this paper have applications in the field of threedimensional visibility. Given a three-dimensional scene, combinatorial changes appearing in the view of a moving observer occur when traversing special surfaces known as visual event surfaces. Such surfaces are swept by lines having prescribed contact with the objects of the scene. Various data structures based on visual events, like the visibility complex or the visibility skeleton [3], have been proposed to speed up visibility computations. The zero-dimensional elements of these structures appear as discrete lines tangent to four objects. Failing to recognize that four objects admit infinitely many tangent lines leads to errors in the computations of these types of data structures. Hence, recognizing configurations of four objects with infinitely many tangent lines is crucial to the robustness of visibility computations. Our theorem settles the case of four spherical objects in $\mathbb{R}^{3}$.

\section{References}

1. C. Borcea, X. Goaoc, S. Lazard, and S. Petitjean. On tangents to quadric surfaces. Preprint, arXiv:math. AG/0402394, 2003.

2. O. Devillers, B. Mourrain, F.P. Preparata, and P. Trebuchet. On circular cylinders by four or five points in space. Discrete Comput. Geom., 29:83-104, 2003. 
3. F. Durand. A multidisciplinary survey of visibility. In ACM SIGGRAPH Course Notes: Visibility, Problems, Techniques, and Applications, 2000.

4. I.G. Macdonald, J. Pach, and T. Theobald. Common tangents to four unit balls in $\mathbb{R}^{3}$. Discrete Comput. Geom., 26:1-17, 2001.

5. G. Megyesi. Lines tangent to four unit spheres with coplanar centers. Discrete Comput. Geom., 26:493-497, 2001.

6. G. Megyesi and F. Sottile. The envelope of lines meeting a fixed line and tangent to two spheres. Discrete Comput. Geom., 33:617-644, 2005.

7. G. Megyesi, F. Sottile, and T. Theobald. Common transversals and tangents to two lines and two quadrics in $\mathbb{P}^{3}$. Discrete Comput. Geom., 30:543-571, 2003.

8. F. Sottile and T. Theobald. Lines tangent to $2 n-2$ spheres in $\mathbb{R}^{n}$. Trans. Amer. Math. Soc., 354(12):48154829,2002

9. T. Theobald. An enumerative geometry framework for algorithmic line problems in $\mathbb{R}^{3}$. SIAM J. Comput., 31(4):1212-1228, 2002.

Received July 13, 2004, and in revised form September 9, 2004. Online publication December 30, 2005. 